\title{
SPONTANEOUS RESOLUTION OF ACUTE SUBDURAL HAEMATOMA
}

Ramandeep Singh Dang1, Deepak S. Panwar², Rajeev Sharma ${ }^{3}$, Sarabjeet Singh ${ }^{4}$, Anil Chandra ${ }^{5}$

\section{HOW TO CITE THIS ARTICLE:}

Ramandeep Singh Dang, Deepak S. Panwar, Rajeev Sharma, Sarabjeet Singh, Anil Chandra. "Spontaneous resolution of acute subdural haematoma". Journal of Evolution of Medical and Dental Sciences 2013; Vol. 2, Issue 45, November 11; Page: 8841-8844.

\begin{abstract}
We report a case of a 47 year old man who presented with head injury due to road traffic accident, with CT scan suggestive of large left sided acute SDH with comminuted fracture of occipital bone. Within a few hours, patient showed significant neurological improvement. Follow-up CT scan revealed rapid spontaneous resolution and redistribution of the acute SDH. The proposed hypothesis explaining this rare phenomenon suggests intracranial and extra cranial redistribution as the cause of spontaneous resolution of acute SDH. The intracranial phenomenon is more common of the two. To our knowledge, this is the first case in the literature where both intracranial and extracranial phenomenon played a role in the same patient leading to rapid spontaneous resolution of acute SDH.
\end{abstract}

KEY WORDS: • acute subdural hematoma • spontaneous resolution • intracranial redistribution • extracranial redistribution.

INTRODUCTION: Acute subdural hematoma (SDH) is a common entity after traumatic brain injury. Acute SDH, more than $10 \mathrm{~mm}$ in thickness, require immediate surgical evacuation in vast majority of the cases. Though a very rare phenomenon, rapid spontaneous resolution of acute SDH does occur $1,2,3,4,5,6,7$. Two possible hypotheses have been suggested: (1) the hematoma is diluted by cerebrospinal fluid (CSF) due to tearing of the arachnoid membrane and then is washed out. (2) The hematoma is compressed by the pressure produced by acute brain swelling and is redistributed 4 . In our current communication, we present a case of spontaneous resolution of acute SDH, the proposed theories explaining spontaneous resolution of acute SDH, as well as, the clinical parameters and imaging characteristics that might predict such a phenomenon are also reviewed. The possibility of spontaneous resolution of an acute SDH, although remote, may affect the decision making process regarding the management of these patients under certain conditions ${ }^{1}$.

CASE REPORT: History: A 47- year- old male patient was admitted in our institute as a case of head injury due to road traffic accident, suffered 1 hour back.

Examination: Post resuscitation, the admission Glasgow Coma Scale (GCS) was E1V1M2. Pupils were bilaterally semi-dilated, measuring $5 \times 5 \mathrm{~mm}$ with sluggish reaction. Computed tomography (C.T) scan on admission revealed an acute SDH over the entire left cerebral hemisphere with a thickness of approximately $22 \mathrm{~mm}$ and a low-density band between the acute SDH and the inner table of the skull. Almost complete obliteration of the contralateral ambient cistern was noted in addition to significant $(17 \mathrm{~mm}$ ) shift of the midline structures to the right. (Image 1) The left lateral ventricle was compressed with dilatation of the right temporal horn of lateral ventricle. There was no evidence of cerebral contusion but diffuse subarachnoid hemorrhage (SAH) and comminuted fracture of occipital bone with overlying sub-galeal hematoma were seen.(Image 2) Basic supportive care, anti-epileptics and cerebral dehydrants were started and tracheostomy done to secure the 
airway. The patient was planned for craniotomy and evacuation of the acute SDH. During preoperative preparation period, the patient showed improvement in GCS score from E1VTM2 to E1VTM5, and surgery was deferred because of this improvement. A repeat CT scan done 11 hours after trauma revealed near complete resolution of acute SDH with a marked decrease in midline shift (12 mm decrease) and visualization of contralateral ambient cistern. (Image 3) An increase in the thickness of sub-galeal hematoma with an increase in the distance between the ends of the comminuted fractured occipital bone was also seen.(Image 4) Seeing this improvement in neuroradiological status, he was continued on conservative management. He showed gradual improvement to a GCS of E3VTM5 over a period of 2 weeks. A repeat CT scan done 2 weeks after admission revealed left hemispheric chronic SDH which was evacuated by double burr hole craniotomy. Postoperatively patient improved further and was discharged in E4V5M6. The patient is in our OPD follow-up since then.

DISCUSSSION: Post-traumatic acute SDH is a life threatening condition. The traditional approach is urgent surgery in hematoma with a mass effect although a conservative therapy is indicated in selected cases. In some of these patients a progressive resolution is appreciated. However a rapid resolution of acute SDH is very rare. The mechanism underlying the rapid resolution of acute SDH is both interesting and somewhat inexplicable. Dural tears or skull fractures may allow the clot to be pushed into the diploic bone of the skull or soft tissue where it may be more rapidly absorbed ${ }^{1}$. The other mechanism may be the tearing of the arachnoid membrane followed by dilution of the hematoma by the cerebrospinal fluid and then is washed out 6 . In some cases, the low density area between the hematoma and the inner wall of the skull was designated as a low density band. The low density band suggests the flow of the CSF into the subdural space, which indicates good outcome for patient with acute SDH ${ }^{1}$. The low density band was also noted in our patient. We considered that this band was related to the mechanism of diminution of SDH in acute stage. The other mechanism proposed is redistribution and dispersal of the hematoma. The redistribution of the acute SDH is usually intracranial. Extracranial redistribution of acute SDH due to fracture or sutural diastasis of overlying skull bone may also occur but is very rare $^{3}$ and only one case is reported in literature till date. In our case there were both low density band and fracture of the skull in the subsequent CT scan. The distance between the ends of the fractured segments was found to be increased with increased thickness of the overlying scalp hematoma thus explaining resolution of acute SDH probably both due to subdural and extracranial redistribution of acute SDH which we feel was natural life saving event in our patient as it lead to resolution of acute SDH with reversal of radiological and clinical signs of brainstem compression as seen in subsequent CT scan.

CONCLUSIONS: Acute subdural hematoma (SDH) is a common entity after traumatic brain injury associated with guarded prognosis and traditionally treated by surgical evacuation. Spontaneous resolution of acute SDH is a rare phenomenon. This study is the first report of a case of spontaneous resolution of posttraumatic acute subdural hematoma due to both intracranial and extracranial redistribution thus affecting the decision making process regarding the management of these patients under certain conditions. 


\section{REFERENCES:}

1. Fujioka Seen, Hamada J, Kaku M, Ushio Y: Rapid resolution of acute subdural hematoma. Report of two cases. Neurol Med Chir (Tokyo) 30: 827-831, 1990.

2. Kapsalaki EZ, Machinis TG, Robinson JS 3 rd, Newman B, Grigorian AA, Fountas KN: Spontaneous resolution of acute cranial subdural hematomas 109 (3): 287-91, 2007.

3. Kundra Surinder N, Kundra Raman: Extracranial redistribution causing rapid spontaneous resolution of acute subdural hematoma. Neuroimage, Neurology India 53: 124-125, 2005.

4. Kunihide Imai: Rapid spontaneous resolution of signs of intracranial herniation due to subdural hematoma. Neurol Med Chir (Tokyo) 43: 125-129, 2003.

5. Noriyuki Kato, Takashi Tsunoda, Akira Matsmura, Kiyoyuki Yanaka, Tadao Nose: Rapid spontaneous resolution of acute subdural hematoma. Neurologica medico-chirurgica -two case reports 41: 140-143, 2001.

6. Matasuyama T, Shimomura T, Okumura Y, Sakaki The: Rapid resolution of symptomatic acute subdural hematoma: case report 48(2): 193-6, 1997.

7. Rivas JJ, Dominguez J, Avilla AP, Martin V, Reyes A: Spontaneous resolution of an acute subdural hematoma. Neurocirugia (Astur) 13 (6): 486-490, 2002.

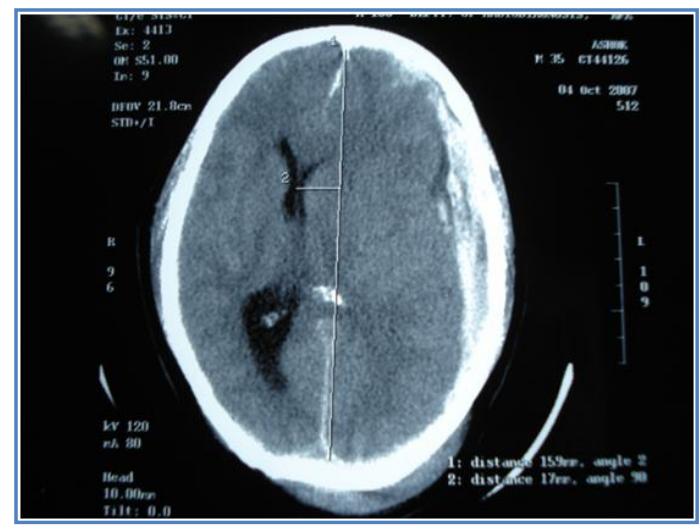

Image 1: Showing left hemispheric acute sub-dural haematoma with midline shift of $17 \mathrm{~mm}$.

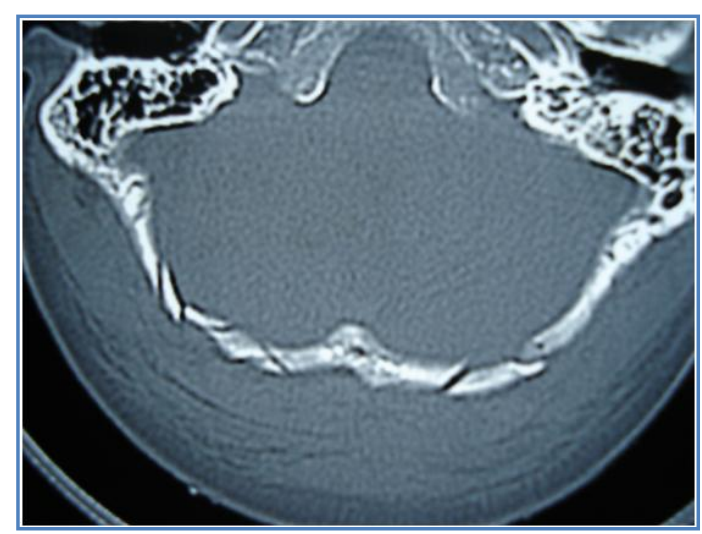

Image 2: Showing comminuted fracture of the occipital bone 


\section{CASE REPORT}

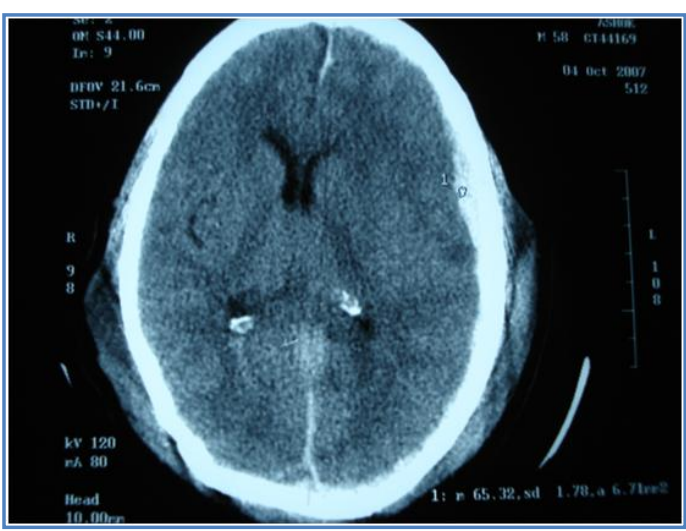

Image 3: Showing near complete resolution of acute SDH with a marked decrease in midline shift (12 mm decrease) and visualization of contralateral ambient cistern.

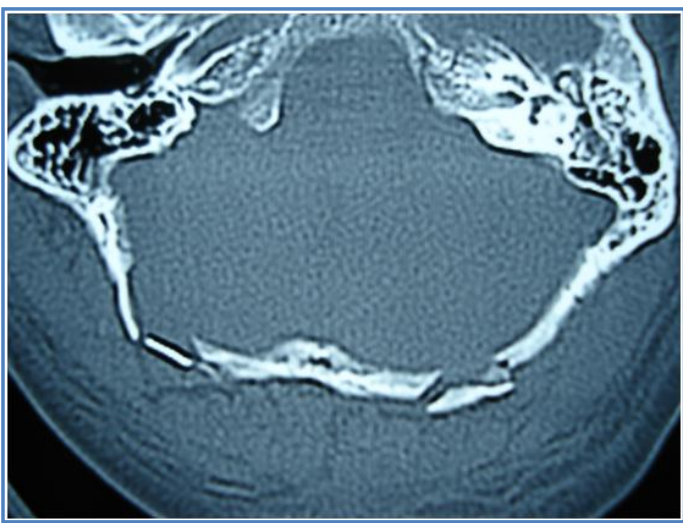

Image 4: Showing increased distance between the ends of comminuted occipital bone fracture ends.

\section{AUTHORS:}

1. Ramandeep Singh Dang

2. Deepak S. Panwar

3. Rajeev Sharma

4. Sarabjeet Singh

5. Anil Chandra

\section{PARTICULARS OF CONTRIBUTORS:}

1. Associate Professor, Department of Neurosurgery, GGSMC, Faridkot.

2. Consultant, Department of Neurosurgery, Haldwani.

3. Associate Professor, Department of Physiology, GGSMC, Faridkot.

4. Assistant Professor, Department of Surgery, GGSMC, Faridkot.
5. Professor, Department of Neurosurgery, KGMU, Lucknow.

\section{NAME ADDRESS EMAIL ID OF THE CORRESPONDING AUTHOR:}

Dr. Ramandeep Singh Dang, Associate Professor,

Department of Neurosurgery, GGSMC, Faridkot.

Email-ramandang@yahoo.com

Date of Submission: 26/10/2013.

Date of Peer Review: 28/10/2013.

Date of Acceptance: 05/11/2013.

Date of Publishing: 07/11/2013 